EPJ Web of Conferences 75, 03006 (2014)

DOI: $10.1051 /$ epjconf/ 20147503006

(c) Owned by the authors, published by EDP Sciences, 2014

\title{
Structural and Electromagnetic Properties of Ni-Mn-Ga Thin Films Deposited on Si Substrates
}

\author{
M. J. Pereira ${ }^{1, a}$, A. A. C. S. Lourenço ${ }^{1}$, V. S. Amaral ${ }^{1}$ \\ ${ }^{1}$ Department of Physics and CICECO, University of Aveiro, 3810-193 Aveiro, Portugal
}

\begin{abstract}
Ni}_{2} \mathrm{MnGa}$ thin films raise great interest due to their properties, which provide them with strong potential for technological applications. $\mathrm{Ni}_{2} \mathrm{MnGa}$ thin films were prepared by r.f. sputtering deposition on $\mathrm{Si}$ substrates at low temperature $\left(400{ }^{\circ} \mathrm{C}\right)$. Film thicknesses in the range $10-120 \mathrm{~nm}$ were obtained. A study of the structural, magnetic and electrical properties of the films is presented. We find that the deposited films show some degree of crystallinity, with coexisting cubic and tetragonal structural phases, the first one being preponderant over the latter, particularly in the thinner films. The films possess soft magnetic properties and their coercivity is thickness dependent in the range $15-200$ Oe at $300 \mathrm{~K}$. Electrical resistivity measurements signal the structural transition and suggest the occurrence of avalanche and return-point memory effects, in temperature cycling through the magnetic/structural transition range.
\end{abstract}

\section{Introduction}

The Ni-Mn-Ga alloy belongs to the class of Heusler alloys that undergo a structural (martensitic) transition involving a transformation in both structural and magnetic properties of the material [1]. This shape memory alloy has become very popular because of its reversible strain induced by a magnetic field [2], a concept called magnetostrain, which was first reported by Ullakko et al. in 1996 [3]. The magnetostrain presented by $\mathrm{Ni}-\mathrm{Mn}-\mathrm{Ga}$ can go up to $10 \%$ and originates from twin boundary motion [1]. Despite the interesting properties that make Ni-Mn-Ga such a popular material, the brittleness of this alloy in the bulk form constitutes a relevant limitation for its use in practical applications. This is one of the reasons that make the study of Ni-Mn$\mathrm{Ga}$ thin films, which are more ductile than the bulk material, extremely relevant. Adding to this, producing this material in the form of thin films allows its application to the development of micro and nano electromechanical systems [4-5]. Most of the research performed so far on the Ni-Mn-Ga system material was focused on the bulk material and only recently some work on Ni-Mn-Ga thin films has been developed. One of the most interesting aspects of the study of Ni-Mn-Ga thin films is the investigation of the role of the substrate in the film's structure and thus in the resulting film properties. Also, the parameters established for the deposition technique are of great relevance for obtaining the desired material properties, as these properties are strongly dependent on the film's composition [6]. Here we investigate the electromagnetic properties of Ni-MnGa films with several thicknesses deposited by radiofrequency (RF) magnetron sputtering onto $\mathrm{Si}(100) / \mathrm{SiO}_{2}$ substrates.

\section{Experimental Details}

$\mathrm{Ni}-\mathrm{Mn}-\mathrm{Ga}$ thin films were deposited onto $\mathrm{Si}(100) / \mathrm{SiO}_{2}$ substrates by radio-frequency (RF) magnetron sputtering. The deposition of the films took place at a substrate temperature of about $400{ }^{\circ} \mathrm{C}$ in an argon atmosphere of less than 10-2 mbar. These conditions are similar to the ones used in [7]. The deposited films possess thicknesses from $10 \mathrm{~nm}$ to $120 \mathrm{~nm}$. The films' thicknesses were obtained through XRR (X-ray reflectivity) and SEM/TEM measurements. The X-ray diffraction measurements were performed on a PANalytical X'Pert PRO MRD X-ray diffraction instrument with a $\mathrm{Cu}-\mathrm{K} \alpha$ radiation source. Isothermal magnetization loops were performed in a Cryogenics VSM (Vibrating Sample Magnetometer) equipment with a resolution of $5 \times 10^{-5}$ emu, whereas magnetization as a function of temperature measurements were performed in a Quantum Design SQUID (Superconducting Quantum Interference Device) magnetometer. The magnetic field was applied in the plane of the films. The chemical composition of the samples was determined by EDS (Energy-dispersive Xray Spectroscopy). Electrical resistivity measurements were performed with a homemade four probe resistivity measurement system.

\footnotetext{
a Corresponding author: mariasapereira@ua.pt
} 


\section{Results and Discussion}

Fig. 1 shows the XRD scans of some of the thinner films, namely the ones with thicknesses of $10 \mathrm{~nm}, 15 \mathrm{~nm}$ and 60 $\mathrm{nm}$, performed at room temperature using grazing incidence configuration in order to suppress the substrate contribution, since these films are quite thin. Fig. 2 shows the XRD scans in the $\theta-2 \theta$ geometry, performed at room temperature, of the Ni-Mn-Ga films with thicknesses of $20 \mathrm{~nm}, 40 \mathrm{~nm}$ and $80 \mathrm{~nm}$.

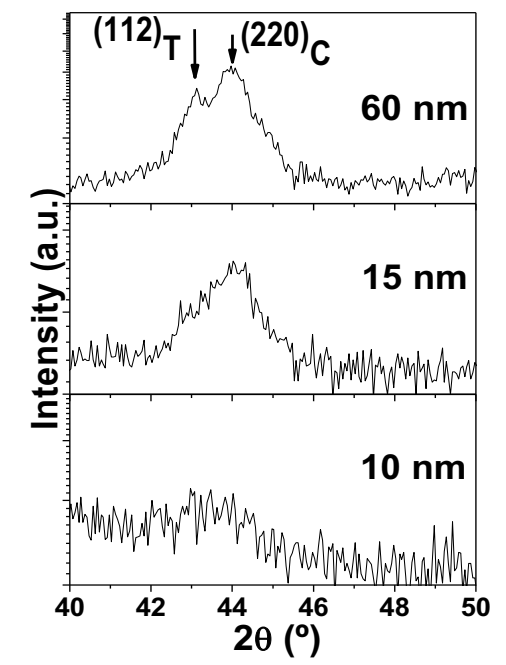

Figure 1. Grazing incidence XRD diffractograms for samples with thicknesses of $10 \mathrm{~nm}, 15 \mathrm{~nm}$ and $10 \mathrm{~nm}$.

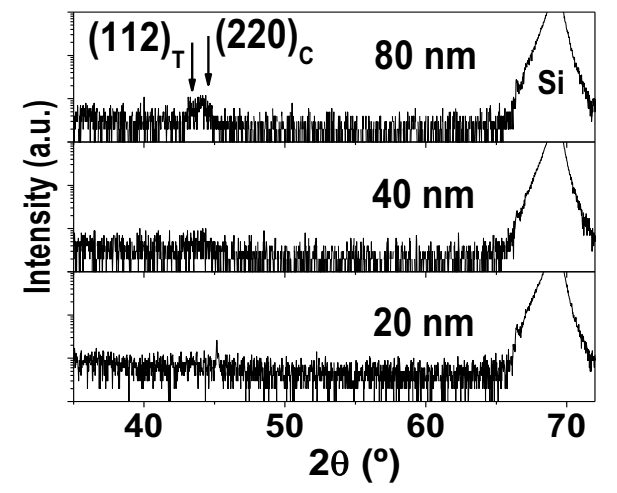

Figure 2. XRD diffractograms for samples with thicknesses of $20 \mathrm{~nm}, 40 \mathrm{~nm}$ and $80 \mathrm{~nm}$.

In fig. 1 it is possible to see that for the $10 \mathrm{~nm}$ film there is almost no noticeable presence of the tetragonal (martensitic) phase and only a contribution from the cubic (austenitic) phase can be perceived. For the $15 \mathrm{~nm}$ film we notice a subtle contribution from the tetragonal coexisting with the cubic phase. The presence of the tetragonal phase becomes even more evident for the 60 $\mathrm{nm}$ film. Fig. 2 confirms the increasing contribution of the tetragonal phase as the film thickness increases. For the $120 \mathrm{~nm}$ film both phases seem to contribute equally for the film's structure. The presence of the $\mathrm{SiO}_{2}$ layer between the substrate and the film has been found to promote the growth of the cubic structure [8]. Fig. 3 a) and c) show surface SEM images of the 120 and $80 \mathrm{~nm}$ films, respectively. Fig. 3 b) and d) show cross section SEM images of the 120 and $80 \mathrm{~nm}$ films, respectively. Fig. 3 e) shows a cross section TEM image of the $60 \mathrm{~nm}$ film.

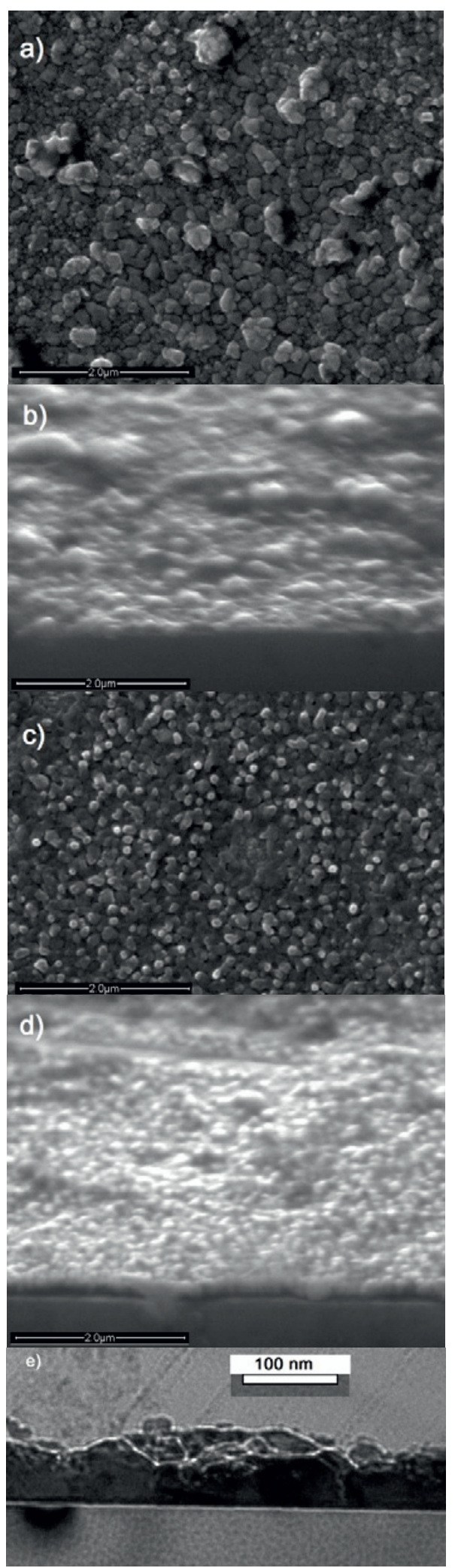

Figure 3. SEM and TEM images: a) b) $80 \mathrm{~nm}$ sample, c) d) 120 nm sample and e) $60 \mathrm{~nm}$ sample. 
We can see that the $80 \mathrm{~nm}$ and $120 \mathrm{~nm}$ samples have an estimated grain size of $0.36 \mu \mathrm{m}$ and $0.2 \mu \mathrm{m}$, respectively. This estimation of the grain size provides information on the roughness and homogeneity of the films' surfaces. For instance, since the $80 \mathrm{~nm}$ film shows bigger grains on its surface than the $120 \mathrm{~nm}$ film, the former is thus expected to possess a higher degree of roughness than the latter. The cross-section image of the $60 \mathrm{~nm}$ film clearly shows the $\mathrm{Si}$ substrate, the $\mathrm{SiO}_{2}$ intermediate layer with a thickness of about $5 \mathrm{~nm}$ and the film with a surface roughness of about $20 \mathrm{~nm}$.

Fig. 4 shows the hysteretic curves of magnetization as a function of the applied magnetic field taken at $300 \mathrm{~K}$ for the films with thicknesses of $20 \mathrm{~nm}, 40 \mathrm{~nm}$ and $80 \mathrm{~nm}$. The analysis of these curves show that at $300 \mathrm{~K}$ the films exhibit soft magnetic properties characterized by narrow hysteresis, low coerciviy and high magnetic saturation values. The saturation magnetization $\left(\mathrm{M}_{\mathrm{S}}\right)$ and coercivity $\left(\mathrm{H}_{\mathrm{C}}\right)$ values obtained for some of the films are listed on Table 1. MS values at $300 \mathrm{~K}$ range from 30 to 200 $\mathrm{emu} / \mathrm{cm}^{3}$. No clear dependence can be established between these values and film thickness. Coercivity $\left(\mathrm{H}_{\mathrm{c}}\right)$ values range from 15 to $200 \mathrm{Oe}$, which can be considered within the same order of magnitude of that of bulk alloys [9]. Fig. 5 shows the coercivity values of the deposited films as a function of film thickness at $300 \mathrm{~K}$. As it can be seen, there is an increase of Hc with thickness, with a clear step between $60-80 \mathrm{~nm}$. This further rise may be correlated with the increase of tetragonal phase in the films thicker than $60 \mathrm{~nm}$. The temperature dependence of the films' magnetization with applied fields of 50, 100 and 2000 Oe was investigated for the samples with thicknesses of 60,80 and $120 \mathrm{~nm}$ in the range of $5 \mathrm{~K}$ to $380 \mathrm{~K}$.

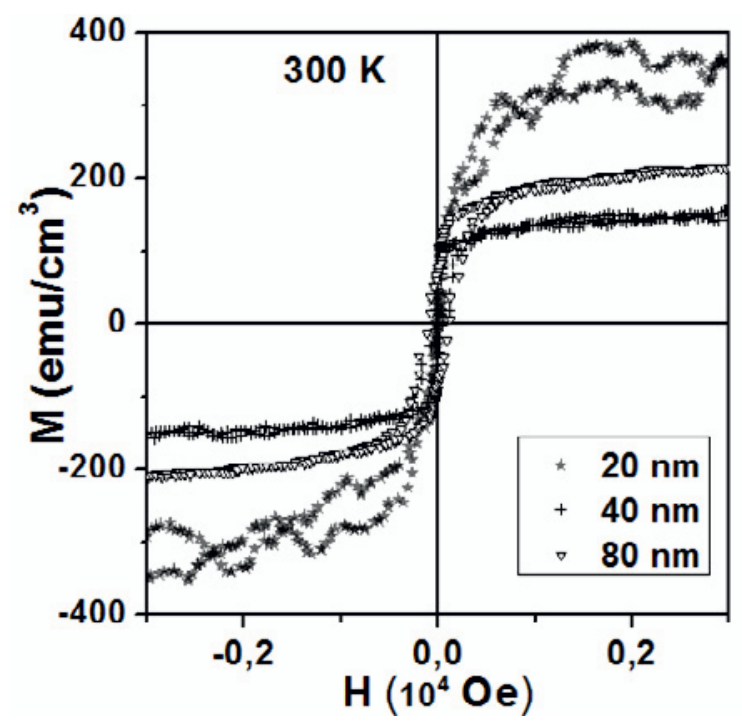

Figure 4. Magnetization as a function of the applied magnetic field taken at $300 \mathrm{~K}$ for the films with thicknesses of $20 \mathrm{~nm}, 40$ $\mathrm{nm}$ and $80 \mathrm{~nm}$.

Fig. 6 shows the corresponding curve obtained for the 60 $\mathrm{nm}$ film, which is representative of the behavior of the analyzed films. The $60 \mathrm{~nm}$ sample displays a $\mathrm{T}_{\mathrm{C}}$ of approximately $350 \mathrm{~K}$, obtained by the extremal derivative $\mathrm{dM} / \mathrm{dT}$. No evidence of first-order structural martensitic to austenitic transition was detected, since no sudden rise in magnetization is noticeable in the temperature range contemplated by the any of the magnetization vs temperature measurements performed. This absence of structural transition evidence may mean that the change in magnetization during the structural transition is too small to be detected in these measurements. The Curie temperature $\left(\mathrm{T}_{\mathrm{C}}\right)$ of the films increases with increasing $\mathrm{Ni}$ content (Table 2), as expected [6].

Table 1. Magnetization saturation and coercivity values at $300 \mathrm{~K}$ for the several film thicknesses.

\begin{tabular}{|c|c|c|c|}
\hline $\begin{array}{c}\text { Temperature } \\
(\mathrm{K})\end{array}$ & $\begin{array}{c}\mathrm{t} \\
(\mathrm{nm})\end{array}$ & $\begin{array}{c}\mathrm{M}_{\mathrm{S}} \\
\left(\mathrm{emu} / \mathrm{cm}^{3}\right)\end{array}$ & $\begin{array}{c}\mathrm{H}_{\mathrm{C}} \\
(\mathrm{Oe})\end{array}$ \\
\hline \multirow{7}{*}{$300 \mathrm{~K}$} & 20 & 300 & 15 \\
\cline { 2 - 4 } & 30 & 30 & - \\
\cline { 2 - 4 } & 40 & 175 & 50 \\
\cline { 2 - 4 } & 60 & 150 & 70 \\
\cline { 2 - 4 } & 80 & 200 & 150 \\
\cline { 2 - 4 } & 120 & 110 & 200 \\
\hline
\end{tabular}

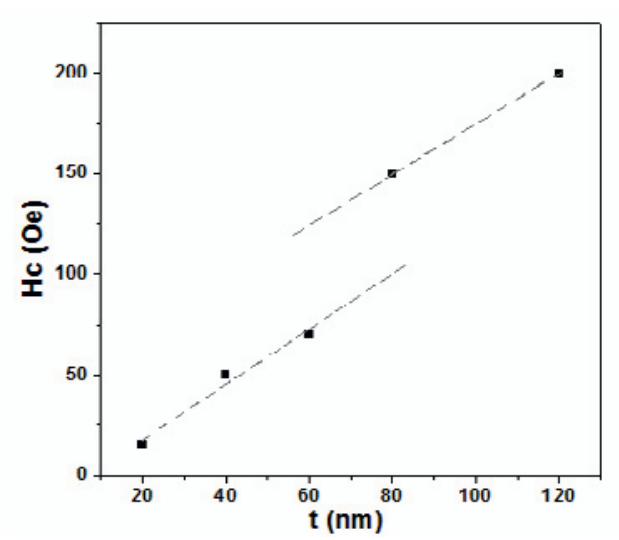

Figure 5. Coercivity values plotted as a function of film thickness.

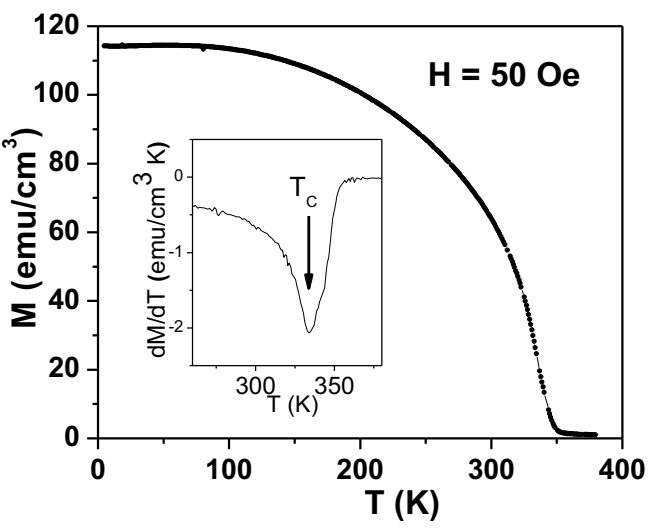

Figure 6. Magnetization versus temperature and corresponding first derivative for the $60 \mathrm{~nm}$ sample. 
Table 2. Film composition and $\mathrm{T}_{\mathrm{C}}$ values for samples with thicknesses of 60,80 and $120 \mathrm{~nm}$.

\begin{tabular}{|c|c|c|c|c|}
\hline $\begin{array}{c}\mathrm{t} \\
(\mathrm{nm})\end{array}$ & $\begin{array}{c}\mathrm{Ni} \\
\text { content } \\
(\%)\end{array}$ & $\begin{array}{c}\mathrm{Mn} \\
\text { content } \\
(\%)\end{array}$ & $\begin{array}{c}\mathrm{Ga} \\
\text { content } \\
(\%)\end{array}$ & $\begin{array}{c}\mathrm{T}_{\mathrm{C}} \\
(\mathrm{K})\end{array}$ \\
\hline 60 & 59 & 27 & 14 & 340 \\
\hline 80 & 53 & 20 & 27 & 316 \\
\hline 120 & 56 & 19 & 26 & 330 \\
\hline
\end{tabular}

Electrical resistance measurements were performed, with particular focus on the transition temperature range. Fig. 7 shows a characteristic resistance versus temperature cycle, $300 \mathrm{~K}$ to $370 \mathrm{~K}$. The jumps appearing at about $340 \mathrm{~K}$ and $330 \mathrm{~K}$ in heating and in cooling respectively, seem to signal the first-order martensitic transformation temperatures. The hysteretic behavior shown by the resistance is typical of shape memory alloys thin films around the martensitic transformation temperature [6]. The structural transition seems to have here, on the dependence of resistance on temperature, a much more pronounced effect than in the magnetization measurements. It is also found that the limits of the temperature cycling modify the smaller details of the curves, suggesting the occurrence of avalanche and return-point memory effects, which will be discussed elsewhere.

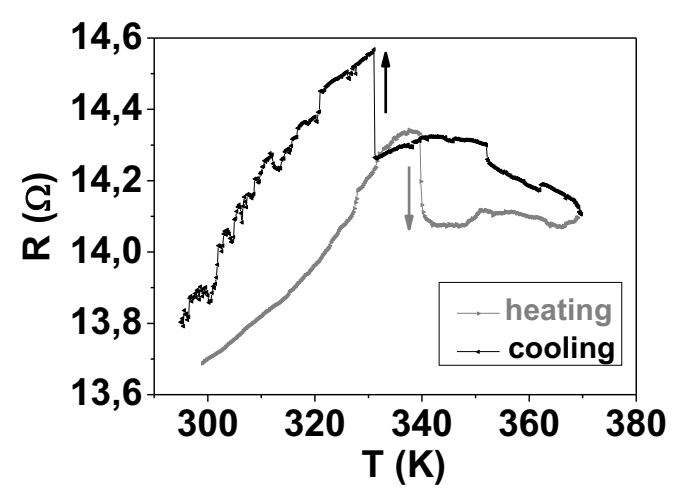

Figure 7. Resistance versus temperature for the $60 \mathrm{~nm}$ sample.

\section{Conclusions}

The studied films show the coexistence of both cubic and tetragonal phases. The cubic phase is preponderant over the tetragonal phase for films with lower thickness values ( $\sim 10 \mathrm{~nm}$ to $60 \mathrm{~nm}$ ) but there is a clear increase of tetragonal phase with film thickness. The films possess ferromagnetic behavior at room temperature and they exhibit soft magnetic properties. Coercivity increases with film thickness. The magnetic transition is clearly observed only in curves of magnetization as a function of temperature. The structural transition has a visible impact on the electrical resistivity changes as a function of temperature on cycling the temperature. These measurements seem to suggest the occurrence of avalanche and return-point memory effects, which are currently under study.

\section{Acknowledgements}

The work is supported by the program COMPETE/ FFEDER and FCT under projects PTDC/CTMNAN/115125/2009 and CICECO - PEstC/CTM/LA0011/2013. M.J. P acknowledges her research grant from FCT project.

\section{References}

1. A. Planes, L. Mañosa, M. Acet, J. Phys. Condens. Matter 21, 233201 (2009)

2. M. Chmielus, X. X. Zhang, C. Witherspoon, D. C. Dunand, P. Müllner, Nature Materials 8, 863-866 (2009)

3. K. Ullakko, J. K. Huang, C. Kantner, R. C. O’Handley, V. V. Kokorin, Appl. Phys. Lett. 69, 1966 (1996)

4. A. Annadurai, A. K. Nandakumar, S. Jayakumar, M. D. Kannan, M. Manivel Raja, S. Bysak, R. Gopalan, V. Chandrasekaran, J. Magn. Magn. Mater. 321, 630634 (2009)

5. M. Thomas, O. Heczko, J. Buschbeck, U. K. Rößler, J. McCord, N. Scheerbaum, L. Schultz, S. Fähler, New J. of Phys. 10, 023040 (2008)

6. V.V. Khovaylo, V.D. Buchelnikov, R. Kainuma, V.V. Koledov, M. Ohtsuka, V.G. Shavrov, T. Takagi, S.V. Taskaev, A.N. Vasiliev, Phys. Rev. B 72, 224408 (2005)

7. M.Y. Teferi et al., Journal of Magnetism and Magnetic Materials 324, 1882-1886 (2012)

8. A. C. Lourenço, F. Figueiras, S. Das, J. S. Amaral, G. N. Kakazei, D. V. Karpinsky, N. Soares, M. Peres, M. J. Pereira, P. B. Tavares, N. Sobolev, V. Amaral, N. M. Santos and A. L. Kholkin, MRS Proceedings 1250-G08-02(2010)

9. I. Babita, R. Gopalan, M. Manivel Raja, V. Chandrasekaran, S. Ram, J. Appl. Phys. 102, 013906 (2007) 